\title{
ANÁLISIS
}

\section{POLÍTICAS DE INFORMACIÓN, BIBLIOTECAS UNIVERSITARIAS Y DESARROLLO: REFLEXIONES DESDE LA COOPERACIÓN UNIVERSITARIA}

\section{Cristóbal Urbano}

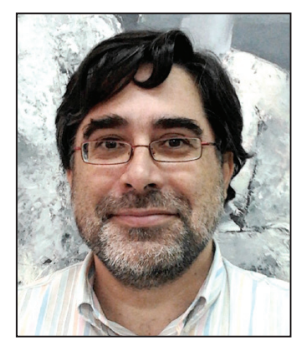

Cristóbal Urbano, profesor titular de la Facultat de Biblioteconomia i Documentació de la Universitat de Barcelona, es miembro del grupo de investigación consolidado i-Viu: información, valor y uso (2009 SGR 846, Generalitat de Catalunya) dedicado entre otras líneas a la evaluación de la investigación en ciencias humanas y sociales, y al estudio del uso de recursos bibliográficos digitales. Su campo de actuación en docencia de grado son los recursos de referencia y los servicios de información. Es profesor de analítica web y de métricas de uso de recursos-e en el Master de gestión de contenidos digitales (UB-UPF). Entre 2010 y 2013 fue el coordinador español del proyecto PCl-Aecid A1/035820/11 de creación del Consorcio de Bibliotecas Universitarias de El Salvador (Cbues). Durante 2013 colaboró con la International Network for the Availability of Scientific Publications (Inasp) en la puesta en marcha de su nuevo plan de acción 2013-2018.

http://orcid.org/0000-0003-0935-6436

Facultat de Biblioteconomia i Documentació, Universitat de Barcelona Melcior de Palau 140, 08014 Barcelona, España urbano@ub.edu

\section{Resumen}

Reflexión sobre la interacción entre políticas nacionales de información y proyectos de cooperación al desarrollo en el campo de las bibliotecas universitarias y de investigación. Se analiza la evolución del concepto políticas de información, su relación con la cooperación al desarrollo, y el papel central que durante casi setenta años ha tenido en la Unesco en dicha dinámica. Finalmente se reflexiona sobre escenarios de cooperación en los que los profesionales de las bibliotecas universitarias y de investigación de países en desarrollo podrían desarrollar proyectos alineados con las políticas nacionales de información.

\section{Palabras clave}

Bibliotecas universitarias, Bibliotecas especializadas, Centros de documentación, Cooperación al desarrollo, Políticas nacionales de información, Políticas públicas, Cooperación interbibliotecaria, Consorcios.

\section{Title: Information policies, university libraries and development: Reflections from university cooperation}

\begin{abstract}
Reflection on the interaction between national information policies and development cooperation projects in the field of academic and research libraries. The evolution of the information policy concept, its relation to development cooperation, and the central role that for nearly seventy years Unesco has played on those matters are analyzed. Finally, cooperation scenarios in which professionals of university and research libraries in developing countries could develop projects aligned with national information policies are examined.
\end{abstract}

\section{Keywords}

University libraries, Special libraries, Documentation centres, Cooperation for development, National information policy, Public policy, Interlibrary cooperation, Consortia.

Urbano, Cristóbal (2014). "Políticas de información, bibliotecas universitarias y desarrollo: reflexiones desde la cooperación universitaria". El profesional de la información, marzo-abril, v. 23, n. 2, pp. 173-182.

http://dx.doi.org/10.3145/epi.2014.mar.10 


\section{Introducción}

La cooperación internacional al desarrollo ha estado muy presente en los últimos años en el ámbito universitario y científico. Por la propia naturaleza de las instituciones académicas la cooperación se vive como una expresión más de la internacionalización por la que mayoritariamente se apuesta hoy en día en la educación superior. Dentro de ese marco más amplio son muchas las instituciones que han participado en proyectos de ayuda al desarrollo fruto de convenios generales entre universidades, convocatorias de agencias nacionales de cooperación o programas estables de organizaciones no gubernamentales de desarrollo (ONGD).

En el caso español el crecimiento de los proyectos de cooperación de las universidades no se puede entender sin el reconocimiento institucional de las autoridades académicas, que cristalizó en 2006 con la creación de la Comisión de Cooperación al Desarrollo de la Conferencia de Rectores de Universidades Españolas (CRUE). Tampoco se puede olvidar el importante apoyo económico y técnico del Programa de Cooperación Interuniversitaria e Investigación Científica $(P C l)$ de la Agencia Española de Cooperación Internacional para el Desarrollo (Aecid) (Moreiro, 2012), las ayudas del cual ni se convocan ni se renuevan desde 2011 a causa del recorte que han experimentado las partidas de cooperación en el presupuesto del Ministerio de Asuntos Exteriores y Cooperación por la crisis económica.

En algunos casos esa colaboración ha comportado intercambio de profesores y alumnos, realización de proyectos de investigación conjuntos, impartición de titulaciones con el aval de universidades extranjeras, actividades de actualización de conocimientos del profesorado o del personal de administración y servicios, así como la puesta en marcha de mejoras en equipamientos universitarios como las bibliotecas. Ahora bien, de forma acertada, una parte importante del apoyo a las bibliotecas universitarias en los proyectos de cooperación se ha vinculado siempre a cubrir necesidades de formación de los profesionales. Por una parte mediante las actividades de capacitación implícitas en los mismos proyectos de desarrollo bibliotecario, por otra mediante programas de posgrado sobre el terreno (Seguí, 1999) o actividades de educación a distancia que han permitido ofrecer cursos de actualización o plazas en las titulaciones oficiales a los profesionales que no pueden cursarlas en sus países (Moreiro, 2012).

La situación de las universidades en los países en desarrollo está marcada por la extensión de la educación básica y media en las últimas décadas, así como por el crecimiento del sector terciario de sus economías y la migración a las ciudades. Todo ello está comportando una mayor demanda social de acceso a la educación superior por parte de capas más amplias de la población, en un gran número con una extracción social más humilde. Por esta razón en muchos países en desarrollo se observa un crecimiento de la iniciativa privada, junto a una saturación y una falta de respuesta de calidad en algunas universidades públicas, lo que ha puesto en la agenda política la necesidad de contar con sistemas de evaluación y aseguramiento de la calidad capaces de regular el sector, incluso con la potestad de autorizar cierres o aperturas de titulaciones y centros.

En ese contexto de evaluación para la mejora de la calidad y la acreditación, el establecimiento de acuerdos con universidades e instituciones extranjeras se ha visto en los países en desarrollo como una oportunidad de obtener recursos materiales y conocimiento para la mejora de la docencia e investigación, pero también para la modernización de la administración y los servicios universitarios. En el caso de los proyectos de cooperación relacionados con las bibliotecas, junto a esa mejora de los recursos materiales, se plantea como un elemento clave el empoderamiento de los bibliotecarios ante sus autoridades académicas y la implicación de éstas en la necesaria integración de las bibliotecas universitarias y de investigación en la agenda de las políticas nacionales de información (PNI).

Los bibliotecarios no han de aceptar que se cuestione su capacidad de influencia por carecer de discurso político sobre la denominada sociedad de la información

\section{Las políticas de información son... ipolítica!}

El término "políticas de información" es muy amplio y difuso, al tiempo que puede formularse a nivel institucional, nacional, regional o internacional. Cuando se contempla a nivel nacional la denominación suele presentarse en singular ("política nacional de información"), pero como concepto suele asociarse a la forma en plural por la propia naturaleza diversa de las acciones, su presentación fragmentaria en ocasiones, los solapamientos y las contradicciones (Hernon; Relyea, 2009, p. 2504). En nuestro caso nos centraremos en estudiar el papel que las bibliotecas universitarias pueden jugar en el conjunto de debates, programas y presupuestos movilizados como "estrategia nacional de información". La reflexión sobre el rol de las bibliotecas se justifica en razón de la evolución que ha tenido el concepto de PNI en las últimas décadas, que ha llevado a formulaciones más amplias a causa del impacto social que tiene el uso extendido de las tecnologías de información y comunicación (TIC) y la globalización (Pajaro-Quesada; Betancourt-Campos, 2007, p. 92). En conjunto se puede decir que la planificación bibliotecaria nacional como un elemento más de estas políticas ha perdido posiciones y eso ha de ser motivo de reflexión para nuestro colectivo profesional (Lor, 2008).

Las PNI se ubican en el ámbito de las políticas públicas y pueden estar representadas por un amplio abanico de actuaciones, desde las que implican una mayor intervención de las instancias de gobierno a las de mera regulación legal. Contemplan tanto la inversión en infraestructura y prestación directa de servicios por parte de los diversos niveles de gobierno (estatal, departamental o local), como la creación de las condiciones adecuadas para que otros agentes participen del proceso alineando sus actuaciones con las metas establecidas a nivel nacional, pasando por el mantenimiento de un marco legal y normativo que permita el ejercicio 
de unos derechos de difusión y acceso universal a la información.

La dimensión ética, administrativa y legal de las PNI se encuentra plenamente de actualidad en razón de la complejidad que la era digital comporta en temas como las concesiones de licencias para la explotación del espacio radioeléctrico, la privacidad, los derechos de autor, la censura y la protección de la imagen y el honor, sin olvidar todo lo relativo al e-gobierno con aspectos clave como el acceso a la información pública, las políticas de transparencia y la protección de los documentos reservados. Para algunos autores como Cornelius (2010, p. 4) el término "políticas de información" se restringe básicamente a estas áreas normativas, sin embargo para la mayoría de autores se ha de considerar una serie amplia de políticas en función del instrumento que se utilice para implementarlas, por lo que podríamos contemplar seis tipos de aproximaciones: "legislación, regulación, construcción de infraestructuras, prestación de servicios, políticas educativas y políticas culturales" (Oppenheim, 1998, p. 47).

En cualquier caso para poder hablar de forma explícita de PNI como conjunto de políticas públicas con entidad propia se han de dar una serie de condiciones: debate social y político en el diagnóstico de la situación y la formulación de las metas, establecimiento de un conjunto de directrices estratégicas, planificación de acciones, identificación de los actores, dotación pública de fondos o estímulo a la movilización de recursos privados.

La existencia de una comisión interministerial, "task-force" o agencia ad hoc de participación público-privada, que articule la coordinación sería otro indicador importante de cara a valorar si se da un planteamiento estratégico de la transversalidad de estas políticas. Dicho órgano coordinador ya se contemplaba como fundamental en las Directrices sobre política nacional de información (Wesley-Tanaskovic, 1985) y en el posterior Manual (Montviloff, 1990) publicados por Unesco, y bajo nombres variados siguen existiendo en la actualidad en los planes nacionales para la "sociedad de la información". En caso que exista un órgano con dichas características a buen seguro los participantes en proyectos de cooperación podrán consultar declaraciones, planes estratégicos, libros blancos o una sede web específica sobre las PNI de cara a ver el lugar que ocupan las bibliotecas universitarias y de investigación, así como la existencia de otros actores con los que se podrían establecer alianzas.

De todas formas el planteamiento holístico que se percibía en las Directrices de la Unesco de 1985 ha quedado en cierta medida superado por la urgencia con la que todos los países han querido subirse al tren de las TIC que lleva a la denominada sociedad de la información y el conocimiento. Desde que en 1993 la administración Clinton-Gore en los Estados Unidos lanzara el proyecto de la National information infrastructure (NII) el foco se ha trasladado desde el término "políticas de información" al de "políticas para la sociedad de la información," algo que se puede explicar a partir del párrafo final del resumen ejecutivo del documento de presentación de la NII: "Una infraestructura de información avanzada permitirá a EUA competir y ganar en la economía global, generando buenos trabajos para los americanos y el crecimiento económico de la nación. Igual de importante será la transformación de las vidas de los americanos -reduciendo las limitaciones en razón del lugar de residencia, de las discapacidades o del estatus económico- pues la NII ofrecerá a todos una oportunidad equitativa para avanzar tan lejos como su talento y ambiciones les lleve" (Information Infrastructure Task Force, 1993).

Esa claridad con la que se presentaban los beneficios perseguidos por la NI/ explica el desencadenante en todo el mundo de una carrera por la competitividad digital de matriz económica, que ha marcado en gran medida la reorientación de las políticas nacionales de información hacia las denominadas "políticas públicas de sociedad de la información" y la implementación de "agendas digitales" (Guerra; Jordán, 2010). Esta evolución quedó sancionada con la celebración de la conferencia World summit on the information society (WSIS) que Naciones Unidas realizó en dos fases (2003 y 2005) y se trasladó a las denominaciones de los ministerios o agencias encargados de estos temas, en ocasiones bajo el mismo paraguas que la investigación y las universidades.

Ahora bien, con independencia del reflejo que estas tendencias hayan tenido en los organigramas de gobierno, en la actualidad se abre paso una visión más realista, fruto de la evaluación de las políticas centradas en el desarrollo de infraestructuras TIC desarrolladas entre 1994 y 2010. Esta nueva etapa ofrece oportunidades para poner las biblio- 
tecas en la agenda como un componente más que puede dotar de contenidos, servicios y dimensión socializadora las TIC. En sus comienzos los planes para "llegar a la sociedad de la información" pusieron el énfasis en el valor casi taumatúrgico de la creación de infraestructura al objeto de evitar la brecha digital entre los países avanzados y los países en desarrollo. Sin embargo las evaluaciones recientes ponen sobre la mesa dos retos: la brecha digital entre los "ricos y los pobres en información" en el interior de los países, y el desarrollo nacional de contenidos y servicios propios que pongan en valor las infraestructuras de acuerdo con las prioridades nacionales. Incluso en el exitoso caso de Corea del Sur, Shin y Kweon (2011, p. 386) alertan de la necesidad de un enfoque más socio-cultural: "Ios gobiernos necesitarán poner más esfuerzo en el aprovechamiento de las fuerzas sociales, e integrar con dichas fuerzas los preparativos tecnológicos para la implantación de la banda ancha como estrategia para un desarrollo progresivo a lo largo del tiempo".

\section{En la Conferencia de Nairobi de 1984} IFLA puso en marcha el programa ALP (Action for development through libraries) que presta un importante servicio a las bibliotecas de países en desarrollo

Sin duda este escenario ofrece nuevas posibilidades que debieran ser tenidas muy en consideración por los bibliotecarios, tanto los del país receptor como los que participan como cooperación exterior, en la formulación de sus proyectos y su capacidad de influir como actores de la formación de políticas públicas en este ámbito. Tal y como titulamos este apartado, las políticas de información son "política" y por ello los bibliotecarios han de evitar asumir el cliché que cuestiona sobre su capacidad de influencia por carecer de discurso político sobre la denominada sociedad de la información. La política no se reduce al discurso, pero sin discurso no hay capacidad de canalizar la participación política. En este sentido no es casualidad que "Libraries on the agenda!" fuera el lema de Claudia Lux como presidenta de la IFLA (2007-2009): respondía a una estrategia de implicarse a nivel político, necesaria tras la pérdida de peso de IFLA en Unesco a partir del año 2000 y la difícil experiencia de visibilizar las bibliotecas en la World summit on the information society (WSIS) promovida por Naciones Unidas (Lor, 2008, 2012).

\section{Desarrollo y políticas de información}

Una rápida mirada a las noticias y las opiniones en prensa pone de manifiesto que en muchas partes del mundo se vive una crisis de representación y participación socio-política, con la que se nos ha hecho pensar que las cosas suceden de forma espontánea al margen de las voluntades políticas. Ante un "Deus ex machina" de alma economicista que parece dirigir la realidad social, resulta más necesaria que nunca la reivindicación de las políticas de información en el marco de la cooperación al desarrollo. Son justamente los países en desarrollo los que con frecuencia están más necesitados tanto de un fortalecimiento de las instituciones democráticas de gobierno como del reconocimiento de las iniciativas colectivas y cooperativas surgidas desde la sociedad civil, por lo que contar con recursos y servicios de información adecuados es a un tiempo un medio para ese fortalecimiento y un objetivo del mismo.

La disponibilidad y el uso de recursos de información constituyen un pilar básico del desarrollo, ya sean considerados instrumentalmente para otras áreas de acción, ya sean vistos en sí mismos como un derecho cultural, de participación ciudadana y de emancipación personal. Pese a que con frecuencia las políticas de información no aparecerán como etiquetas principales de los objetivos urgentes de las políticas de desarrollo, su presencia es transversal, instrumental y a más largo plazo.

La generación, captación y uso del conocimiento es fundamental para un desarrollo sostenible, por ello entre los organismos de ayuda al desarrollo se considera clave este aspecto de cara a consolidar las mejoras. Como ejemplo podemos tomar la referencia de los Objetivos de desarrollo derivados de la Declaración del Milenio (ODM) (Naciones Unidas. Asamblea General, 2000) y como en algunos trabajos se ponen en relación con las políticas de información. Un primer caso podría ser el del Netherlands Development Assistance Research Council (2005, p. 9), que señalaba como un reto para la consecución de los objetivos "la ausencia de capacidades suficientes para el desarrollo basado en el conocimiento en países destinatarios de la cooperación". Por su parte Andrew Kaniki (2008, p. 16), al estudiar el papel de las bibliotecas africanas y la ODM remite a la presencia de la información en todo el ciclo de las actuaciones de desarrollo, destacando que al margen de la intervención inicial, las actuaciones "han de generar localmente nuevo conocimiento y recursos humanos capacitados en usarlo de forma recurrente".

A la vista de esta evidencia, resulta claro que los países en desarrollo necesitarán políticas activas de información que permitan el establecimiento de infraestructuras, la prestación de servicios, la adquisición y generación de recursos de información y la educación de los agentes y los usuarios. Esto es, no será posible pensar únicamente en políticas de intervención basadas simplemente en dotación de infraestructuras TIC, o en ceder el campo de actuación en su totalidad a los agentes privados, bajo el mero establecimiento de unas normas legales reguladoras de derechos individuales.

Dado que la información atraviesa horizontalmente un buen número de actividades sociales, culturales y políticas, los PNIs deberían contemplarse como una política de estado, que precisa de periodos de desarrollo superiores a los de un gobierno concreto para su consolidación y el logro de sus fines (Pajaro-Quesada; Betancourt-Campos, 2007, p. 89). Esto no significa que el estado lo tenga que hacer todo, pero implica crear las condiciones para que la participación empresarial o la de las instituciones no lucrativas puedan contribuir a unos objetivos colectivos. Al igual que sucede en otros entornos como la educación, en las PNI la política partidaria de corto alcance es un riesgo para el desarrollo sostenible. 


\section{La trayectoria de Unesco y otras organizaciones internacionales}

Para los países en desarrollo siempre ha sido muy importante el apoyo material, pero también el asesoramiento técnico de organizaciones internacionales, especialmente aquellas pertenecientes al Sistema de Naciones Unidas. También han jugado un papel relevante las ONGDs de amplio alcance internacional y trayectoria contrastada. Por eso todo proyecto universitario de cooperación debería tener en consideración las directrices de dichas organizaciones y explorar su trayectoria en el país.

Como punto débil habría que apuntar que en ocasiones las acciones de organismos internacionales en relación con las PNI adolecen de poca implicación de los agentes no gubernamentales en la formación de dichas políticas. Con frecuencia las actuaciones consisten en facilitar la participación de representantes de gobiernos en reuniones y cumbres diversas, que posteriormente casi no tienen traducción en la agenda política interna, sin que se generen las alianzas necesarias con los actores sobre el terreno. Otro punto débil sería el de la burocratización de los órganos y procedimientos establecidos en algunos países en desarrollo en respuesta a las recomendaciones publicadas en directrices internacionales. En general se puede constatar que planteadas las políticas desde "arriba" con frecuencia superan la fase del informe o del libro blanco, de la comisión parlamentaria, de la campaña política o mediática, pero si no van dotadas de medios o no crean sinergias con las instituciones y profesionales implicados, su impacto acostumbra a ser mínimo. De todas formas, los documentos y los programas que proponen recomendaciones internacionales, las buenas prácticas o los estudios de prospectiva, son muy valiosos como discurso de respaldo a los procesos de formación de políticas "desde abajo".

En tanto que organización de Naciones Unidas especializada en educación, ciencia y cultura, la Unesco ha sido responsable en buena medida de la internacionalización del concepto "políticas de información". Desde su fundación, sus documentos y proyectos han apoyado a los países en desarrollo en la extensión de la alfabetización, la libertad de prensa e información, o las bibliotecas. El Manifiesto de la biblioteca pública de 1947 y su posterior revisión (Unes$c o, 1994)$ son un buen ejemplo de una primera etapa en la que sus documentos han tenido una gran influencia en la conformación de políticas nacionales vinculadas a cultura y educación como elementos basales de lo que hoy denominamos políticas de información en sentido amplio.

En una segunda etapa Unesco situó el foco en primera instancia en la información científico-técnica, para posteriormente ampliarlo a la información especializada, académica y profesional en todos los ámbitos del conocimiento. La celebración en París de la Conferencia intergubernamental para el establecimiento de un sistema mundial de información científica (Unisist) (1971) derivó en 1972 en la creación de Unisist como programa estable de la organización con el objetivo de fomentar la transferencia de información científica para el desarrollo económico y social mediante una acción internacional coordinada y sostenida (Soler, 2007, p. 81). El programa requería la articulación de redes internacionales de bases de datos especializadas bibliográficas y factuales, a las que se debería acceder y contribuir desde todos los países independientemente de su nivel de desarrollo. Este objetivo puso sobre la mesa la necesidad de "un organismo gubernamental o semi-gubernamental en el plano nacional encargado de guiar, estimular y coordinar el desarrollo de los recursos y servicios de información en la perspectiva de la cooperación nacional, regional e internacional" (WesleyTanaskovic, 1985, p. i).

Lógicamente para alcanzar dicho objetivo Unesco estimuló la cooperación y el trabajo en red de bibliotecas especializadas y centros de documentación, al tiempo que ayudó a la creación de sistemas nacionales de información mediante misiones de asistencia, documentos para normalizar los procesos técnicos y directrices sobre el desarrollo de las políticas de información.

La generación, captación y uso del conocimiento es clave para un desarrollo sostenible

De esta segunda etapa aún podemos observar realizaciones activas muy importantes para los países en desarrollo y para algunos de los que hoy consideramos emergentes.

Un primer ejemplo sería la distribución desde 1985 de CDS/ $I S I S$, software gratuito para la gestión de bases de datos bibliográficas, líder en muchos países como solución de informática documental hasta la aparición de programas open source a finales de los años 90.

En segundo lugar podemos mencionar la creación de bases de datos especializadas mediante la colaboración internacional, como sería el caso de la producción científica de ciencias de la salud en América Latina con Lilacs, elaborada por Biblioteca Regional de Medicina (Bireme) de la Organización Panamericana de la Salud. Los sistemas de información de la $F A O$ son otro de los ejemplos destacados para ilustrar el movimiento puesto en marcha por Unisist, ya sea por su apoyo a la creación de redes de bibliotecas agrícolas como Aglinet, la publicación de bases de datos de matriz cooperativa como Agris, o la difusión de normas y buenas prácticas para el desarrollo de sistemas abiertos de información agrícola con Aims.

http://lilacs.bvsalud.org

http://www.fao.org/library/library-home/aglinet

http://agris.fao.org

http://aims.fao.org

Finalmente podríamos considerar una tercera etapa, fruto de las transformaciones asociadas al desarrollo mundial de internet. Dichas transformaciones agudizaron las contradicciones y evidenciaron los resultados desiguales del Programa general de información (PGI) y del Programa intergubernamental de informática (PII) con los que en 1976 se amplió el alcance del Unisist. En 2000 el PGI y el PII se fusionaron y dieron lugar a IFAP (Information for all programme) para dar respuesta a la era internet y a la globalización digital, 


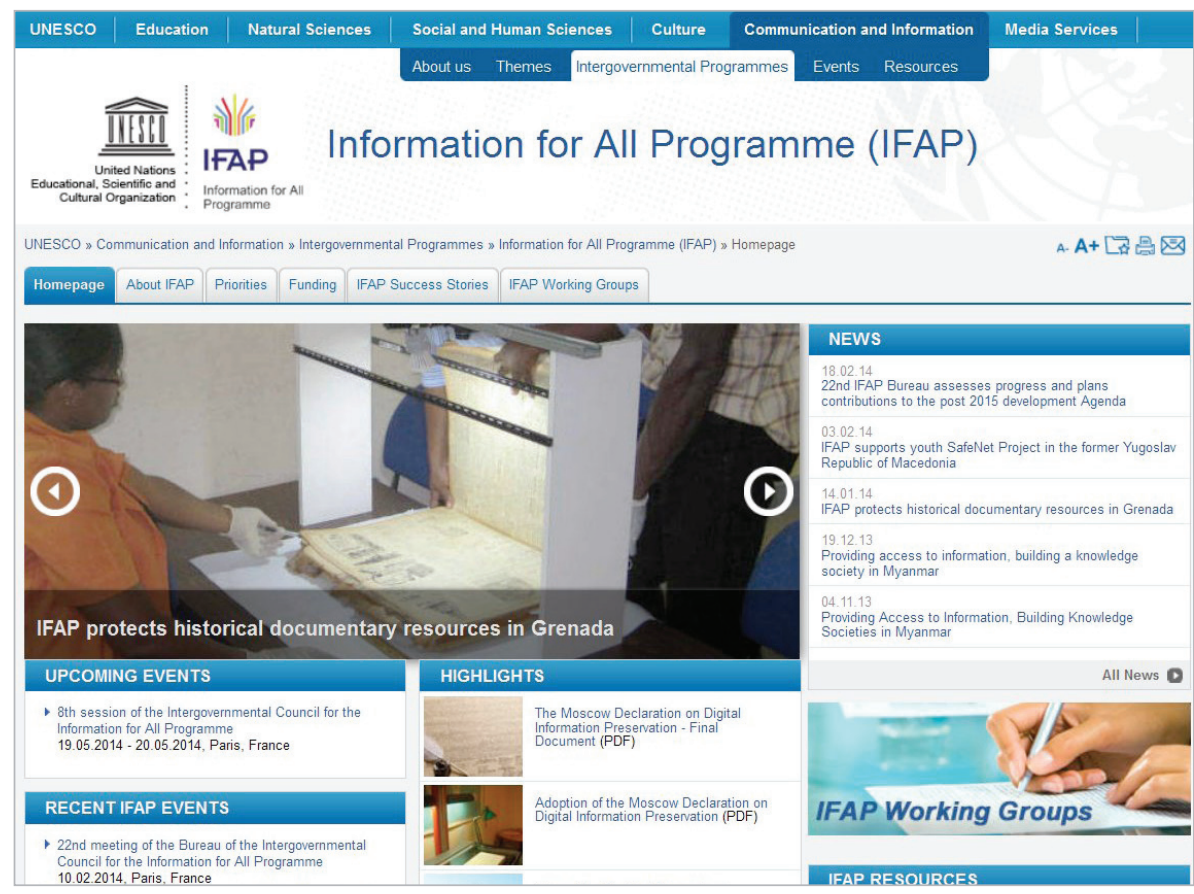

http://goo.gl/Gv1q7G ayudando a los estados miembros de Unesco a formular las nuevas políticas nacionales de información derivados de los progresos en el uso de las TIC y evaluar los retos que presenta el tránsito a la denominada "sociedad del conocimiento" (Bindé, 2005).

Esta nueva etapa supone en buena medida la adopción por parte de Unesco de un discurso similar a los que a nivel nacional se desencadenaron en 1993 con la NII de Estados Unidos y que a nivel internacional lidera la UIT (Unión Internacional de Telecomunicaciones), organismo especializado de las Naciones Unidas para las tecnologías de la información y la comunicación. Todo ese movimiento culmina con el importante papel de Unesco en 2003 y 2005 en la organización de la citada WSIS y en el seguimiento del conjunto de resoluciones de la conferencia que se le asignaron (Souter, 2005).

\section{http://www.itu.int/wsis/basic/index.html}

El resultado de toda esa evolución es el de una organización más preocupada por los "media" y las TIC como elementos tractores de la transformación de las políticas nacionales de información, que no manifiesta de forma suficientemente explícita que las bibliotecas y los servicios de información científica también han de ser parte de esa transformación. En este sentido es interesante la posición de Lor (2008, p. 41) quien como secretario general de IFLA entre 2005 y 2008 representó a IFLA en la WSIS: “...es decepcionante lo poco que se dice en la Estrategia a medio plazo de la Unesco sobre las bibliotecas como agentes centrales en las sociedades del conocimiento del futuro. Cuando se mencionan las bibliotecas se hace de forma accesoria. El único momento en el que la palabra biblioteca genera algún tipo de entusiasmo es cuando se mencionan las bibliotecas digitales".

Junto al papel fundamental de Unesco en el debate de las políticas de información, han de ser tomadas en consideración diversas organizaciones profesionales y ONGDs. Se ha de mencionar el papel de IFLA, especialmente a partir de 1984 cuando en la Conferencia de Nairobi se puso en marcha el programa ALP (Action for development through libraries) que presta un importante servicio a las bibliotecas de países en desarrollo mediante el intercambio profesional, el fortalecimiento de las asociaciones nacionales de bibliotecarios, la difusión de pautas y buenas prácticas para la implementación de los servicios bibliotecarios, o su labor como grupo de presión en foros internacionales para que se consideren las bibliotecas como un instrumento de futuro en la promoción del desarrollo. Especialmente relevante en este sentido es su reciente Statement on libraries and development (IFLA, 2013). http://www.ifla.org/alp

Por último en el campo de las bibliotecas universitarias y de investigación no se puede entender la mejora en accesibilidad a la bibliografía científica en todo el mundo sin los recursos-e ofrecidos por Research4Life, programa promovido por la Organización Mundial de la Salud, o sin el trabajo integral de ONGDs como Inasp o eFIL que promueven el fortalecimiento de estructuras nacionales y consorcios para el desarrollo cooperativo de colecciones, servicios bibliotecarios y plataformas de edición electrónica.

http://www.research4life.org

http://www.inasp.info

http://www.eifl.net

Estas iniciativas han hecho posible que prácticamente cualquier investigador o docente universitario de un país en desarrollo cuente con acceso online a un stock de información científica de primer nivel, equivalente en lo fundamental a la que pudiera tener un colega del primer mundo. Al tiempo que con estos programas se amplía la oferta de recursos de información, se trabaja para superar las barreras al uso de información de calidad:

- ampliando la base de usuarios con el alumnado universitario y los profesionales;

- optimizando la gestión de las redes informáticas y los puntos de consulta;

- mejorando la organización y catalogación de los e-recursos;

- mejorando la capacitación de los bibliotecarios; y

- haciendo de la alfabetización informacional una actividad significativa en el contexto del cambio hacia modelos docentes centrados en el alumno (Urbano, 2013). 


\section{Escenarios para las bibliotecas universitarias y de investigación}

Pese a las limitaciones materiales, en muchos países en desarrollo las universidades son instituciones de prestigio y bajo su relativa autonomía política con frecuencia pueden mantener un compromiso social y cultural con una cierta estabilidad institucional que no se da en otras instituciones. Eso explica que en no pocos casos realicen tareas de suplencia en materia por ejemplo de patrimonio cultural, o que el sistema universitario en su conjunto ejerza un papel fundamental en la recolección, transformación y suministro de información del país. Esas circunstancias y su capacidad de cooperación exterior facilitan que en el sector de las bibliotecas universitarias se puedan desarrollar proyectos que actúen como elemento tractor para otros sectores del país, por ejemplo mediante la introducción y aprendizaje de procesos innovadores o transformadores.

\subsection{Trabajar juntos, aprender juntos: consorcios y redes}

El eje principal en la formación de PNI relacionadas con el tipo de bibliotecas que nos ocupa sería la consideración del conjunto de centros como elementos que han de cooperar lo más intensamente posible para alcanzar sinergias y economías de escala. El trabajo cooperativo, liderado o apoyado desde instancias políticas nacionales, es fundamental en todos los ámbitos que consideramos como oportunidades o retos para estas bibliotecas, pero especialmente lo es en tanto que ayuda a la ruptura del aislamiento profesional de los bibliotecarios -entre ellos mismos y respecto a la sociedad- y a la mejora de su capacitación profesional. El gran potencial de las actuaciones que abunden en esta línea se visualiza claramente en el título que hemos dado a este apartado "trabajar juntos, aprender juntos", inspirado en un artículo en el que se presentaban los primeros años de trayectoria del Consorci de Biblioteques Universitàries de Catalunya (Anglada, 1999).

La creación de un programa de formación continuada para la elevación de las competencias y los conocimientos de los profesionales puede considerarse una de las principales justificaciones del trabajo cooperativo y de la creación de redes o consorcios. La mejora del perfil profesional de los bibliotecarios, la participación de otros profesionales en el impulso de las bibliotecas universitarias y la decantación de liderazgos como portavoces de las redes o consorcios puede facilitar la consideración de los profesionales de las bibliotecas como actores en la formación de PNI en pie de igualdad con otros sectores.
Como ya hemos visto las políticas de información no se vinculan únicamente a políticas públicas elaboradas e implementadas desde las instancias de gobierno, sino como la coordinación de las políticas de los diversos actores en materia de información de un país bajo un marco adecuado para su apoyo y reconocimiento como partícipes de un plan nacional. Como buena parte de las bibliotecas que nos ocupan pueden tener una débil vinculación orgánica con el gobierno, o pueden ser totalmente independientes del mismo, su interlocución será mucho más eficaz si forman parte de una red, asociación o consorcio. Tomar decisiones de política nacional no siempre comporta dedicar recursos económicos, materiales o humanos, sino que puede consistir en empoderar a los que puedan llevar a mejor término unas determinadas acciones, dentro de una cierta visión nacional de conjunto que se consigue entrando en relación con los profesionales de las bibliotecas de otras instituciones.

La participación en redes nacionales e internacionales, el fortalecimiento de estructuras cooperativas, la creación de consorcios de bibliotecas, serían ejemplos en el plano institucional que pueden dotar de contenido al concepto "trabajar juntos" como meta de los proyectos de cooperación. En el plano de la mejora de los recursos y de los servicios, entre otros, podríamos hablar de plataformas conjuntas de edición electrónica, proyectos de digitalización retrospectiva, elaboración de programas y materiales de calidad para la alfabetización informacional, políticas de preservación digital, repositorios de investigación y de gestión de datos o desarrollo cooperativo de colecciones.

Cualquiera de esos ejemplos de mejora en servicios y recursos precisa del fortalecimiento institucional y de la interacción política para que se pueda hablar de sostenibilidad. Por esa razón Inasp, ONGD que facilita el acceso a recursos-e de información científica y apoya la creación de consorcios en 22 países, ha elegido para su nuevo plan de actuación 
2013-2018 el lema Strengthening research and knowledge systems, en el que la palabra "sistema" apela a ese respaldo político e institucional a escala nacional.

http://www.inasp.info/en/work/what-we-do/programmes/srks

\subsection{Reflexionar juntos, para encontrar un camino propio}

Buena parte del análisis de escenarios para proyectos de cooperación podría beber de multitud de artículos e informes publicados en los últimos tiempos sobre cómo se ha de afrontar el futuro las bibliotecas universitarias. Sin duda hay retos compartidos con bibliotecas de los países con mayor desarrollo bibliotecario. Los escenarios prospectivos que se dibujan para este tipo de bibliotecas en aquellos países que durante el último siglo han liderado la evolución en este campo son complejos y exigirán algo más que una evolución adaptativa como la que se ha experimentado hasta ahora (ACRL Research Planning and Review Committee, 2012, 2013; Anglada, 2012; Lewis, 2013). Es pues importante estar muy al día de los estudios que analizan tendencias y buenas prácticas de innovación, pero realizando una lectura "dialogada" con la realidad sobre el terreno, desde la experiencia de los profesionales y autoridades académicas del país de destino de la cooperación. El resultado de esa reflexión puede tener un impacto positivo en el cuestionamiento de las rutinas propias del país de procedencia de la cooperación. Potenciar las habilidades de evaluación y de planificación entre todos los participantes ha de ser en sí mismo un objetivo prioritario.

La primera condición para influir en la agenda de las políticas de información es que los propios bibliotecarios se crean su capacidad de influencia y asuman el compromiso de ejercerla

\subsection{Visibilizar las bibliotecas y sus profesionales}

La primera condición para influir en la agenda de las políticas de información es que los propios bibliotecarios se crean su capacidad de influencia y asuman el compromiso de ejercerla. Esta idea se ilustra bien con la situación descrita por Ayoo y Otike (2002, p. 354) en referencia a los bibliotecarios en Kenia pero es extrapolable a muchos otros sitios: "falta compromiso de los profesionales en la reivindicación de las mejoras de las infraestructuras de información o de la profesión". Por tanto, las acciones de cooperación exterior deben ayudar a situar a los bibliotecarios de estos centros como actores de sus políticas nacionales, tanto en su condición de implementadores de base como en la de generadores de opinión e influencia en la conformación de las mismas. La elaboración de un diagnóstico previo a la redacción del proyecto de cooperación y la interacción con las autoridades para informar de los objetivos del mismo serían espacios propicios para el análisis de las políticas nacionales y para la concienciación de los profesionales de su papel.

La falta de visibilidad del mundo bibliotecario en el nuevo discurso de las políticas nacionales de información ha de ser motivo de reflexión más que de lamento y exige una respuesta que no consista en dar la espalda a los signos de los tiempos. Especialmente en proyectos de cooperación al desarrollo uno de los resultados ha de ser que los bibliotecarios logren hacerse visibles en las plataformas en las que se formulan políticas de información, leyendo como oportunidad y en clave bibliotecaria las líneas de actuación emanadas por ejemplo de la WSIS o de la agenda digital de su gobierno. Lor (2012, p. 279) ofrece unas recomendaciones muy claras en este sentido: "Los bibliotecarios deberían elevar la sensibilización en el seno de Unesco sobre el valor y el papel de las bibliotecas mediante la participación en su comisión nacional del programa IFAP. Si dicho comité no existe aún, deberían ayudar a establecerlo con la ayuda de otros miembros de IFLA de su país".

Por último pero no menos importante, la capacidad de comunicar a los usuarios los servicios y recursos que la biblioteca les ofrece y de establecer con ellos una vinculación de compromiso mutuo es en general un punto débil de muchas bibliotecas, lo que en ocasiones está asociado a las dificultades para demostrar el retorno de los recursos dedicados (McCreadie, 2013, p. 23). Estudiar la satisfacción de los usuarios, sus necesidades, sugerencias y quejas representa un primer nivel de relaciones públicas, de evaluación del desempeño y de revisión de políticas a nivel de cada institución que se tiene que dar en todo proyecto de mejora, también en los de cooperación internacional. La visibilidad se ha de trabajar en primer lugar con los propios usuarios, reales o potenciales.

\subsection{Tener voz propia en la Red}

Todos los países tienen el derecho a ofrecer una visión científica de su propia realidad, sin depender totalmente de la información procedente del exterior que es fácilmente accesible en internet. También tienen derecho a que la imagen digital de su patrimonio cultural no dependa en exclusiva del exterior. La generación endógena de desarrollo requiere de la existencia en cada país de una base de pensamiento, de evidencias científicas, de información y de un relato cultural propio que no margine el conocimiento tradicional, pero que no se puede alimentar sin universidades e institutos de investigación que cuenten con servicios bibliotecarios y de información adecuados.

\section{Conclusiones}

En los proyectos de bibliotecas universitarias y de investigación realizados con cooperación exterior entran en juego diversos actores públicos y privados, nacionales y extranjeros, pero la acción se produce bajo el marco político nacional del país receptor, por lo que resulta fundamental el conocimiento de las políticas nacionales de información (PNIs) y su presencia transversal en las políticas educativas, culturales y de investigación.

La sostenibilidad de las acciones de cooperación en bibliotecas y su adecuación al contexto será siempre mayor si se da este conocimiento entre los profesionales nacionales y los cooperantes extranjeros. Tanto si existe un cuerpo de políticas bien establecidas como si su ausencia requiere un trabajo de "abajo a arriba" para su articulación como producto 
del propio proyecto de cooperación, habrá que considerar convenientemente la trayectoria nacional, los referentes de otros países y las recomendaciones internacionales en políticas de información.

El fortalecimiento de los sistemas nacionales de información y de conocimiento pasa en muy buena medida por la creación de consorcios de bibliotecas y estructuras de cooperación para la creación de contenidos digitales. La priorización de los recursos de las agencias de cooperación al desarrollo tendría que tener en consideración que este enfoque sistémico está avalado por las políticas de los organismos internacionales y de las ONGDs especializadas en bibliotecas.

\section{Bibliografía}

ACRL Research Planning and Review Committee (2012). "2012 top ten trends in academic libraries". College \& research libraries news, v. 73, n. 6, pp. 311-320.

http://crln.acrl.org/content/73/6/311.full.pdf+html

ACRL Research Planning and Review Committee (2013). Environmental scan 2013 (45 pp.). Chicago: ALA.

http://www.ala.org/acrl/sites/ala.org.acrl/files/content/ publications/whitepapers/EnvironmentalScan13.pdf

Anglada, Lluís (1999). "Working together, learning together: the consortium of academic libraries of Catalonia". Information technology and libraries, v. 18, n. 3, pp. 139-144.

Anglada, Lluís (2012). "Bibliotecas universitarias: cabalgando la tecnología, siguiendo al usuario". El profesional de la información, v. 21, n. 6, pp. 553-556.

http://www.elprofesionaldelainformacion.com/contenidos/2012/ noviembre/01.pdf

http://dx.doi.org/10.3145/epi.2012.nov.01

Ayoo, Philip; Otike, Japhet (2002). "Factors hampering the formulation of a national information policy in Kenya". Library review, v. 51, n. 7, pp. 350-357.

http://dx.doi.org/10.1108/00242530210438646

Bindé, Jérôme (2005). Hacia las sociedades del conocimiento: informe mundial de la Unesco. Paris: Unesco. ISBN: 923 3040003

http://unesdoc.unesco.org/images/0014/001419/141908s.pdf

Cornelius, Ian (2010). Information policies and strategies. London: Facet. ISBN: 9781856046770

Guerra, Massiel; Jordán, Valeria (2010). Políticas públicas de sociedad de la información en América Latina: ¿una misma visión? Santiago de Chile: Comision Económica para América Latina y el Caribe (Cepal).

http://www.eclac.cl/ddpe/publicaciones/xml/1/39181/ W314Esp.pdf

Hernon, Peter; Relyea, Harold C. (2009). "Information policy: United States". En: Encyclopedia of Library and Information Sciences. 3rd ed. New York: Taylor \& Francis. p. 2504-2518.

http://www.tandfonline.com/doi/abs/10.1081/EELIS3-120009040

IFLA (2013). IFLA statement on libraries and development. http://www.ifla.org/publications/ifla-statement-onlibraries-and-development
Information Infrastructure Task Force. United States (1993). The National information infrastructure: agenda for action. Washington DC: Executive Office of the President. http://files.eric.ed.gov/fulltext/ED364215.pdf

Kaniki, Andrew (2008). "Libraries and information services in facilitating knowledge production and management for the Millennium Development Goals". En: Njobvu, Benson; Koopman, Sjoerd (Eds.). Libraries and information services towards the attainment of the UN Millennium Development Goals. München: K. G. Saur, pp. 15-27.

http://www.degruyter.com/viewbooktoc/product/40598

Lewis, David W. (2013). "From stacks to the Web : the transformation of academic library collecting". College and research libraries, v. 74, n. 2, pp. 159-177.

http://crl.acrl.org/content/74/2/159.full.pdf

Lor, Peter-Johan (2008). “MDGS, WSIS, Unesco's MTS and IFAP: alphabet soup or opportunities for libraries?". En: Njobvu, Benson; Koopman, Sjoerd (Eds.). Libraries and information services towards the attainment of the UN Millennium Development Goals. München: K. G. Saur, pp. 29-55. http://www.degruyter.com/viewbooktoc/product/40598

Lor, Peter-Johan (2012). "The IFLA-Unesco partnership 1947-2012". IFLA Journal, v. 38, n. 4, pp. 269-282.

http://www.ifla.org/files/assets/hq/publications/ifla-journal/ifla-journal-38-4_2012.pdf

http://dx.doi.org/10.1177/0340035212463138

McCreadie, Nell (2013). Library value in the developing world. Sage.

http://www.uk.sagepub.com/repository/binaries/pdf/ LibValReport-2013.pdf

Montviloff, Victor (1990). Políticas nacionales de información: manual sobre la formulación, aprobación, aplicación y funcionamiento de una política nacional sobre la información. Paris: Unesco.

http://unesdoc.unesco.org/images/0008/000869/086995sb. $p d f$

Moreiro, José-Antonio (2012). “Docencia e investigación universitarias en información y documentación para la cooperación al desarrollo". Anuario ThinkEPI, v. 6, pp. 31-34. https://payhip.com/b/Ut/4

Naciones Unidas. Asamblea General (2000). Declaración del Milenio.

http://www.un.org/spanish/milenio/ares552.pdf

Netherlands Development Assistance Research Council (2005). Mobilizing knowledge to achieve the millennium development goals. The Hague: Rawoo.

http://docs.watsan.net/Downloaded_Files/PDF/Rawoo2005-Mobilizing.pdf

Oppenheim, Charles (1998). "Current UK and EU information policy". En: Grieves, Maureen (ed.). Information policy in the electronic age. London: Bowker-Saur, pp. 45-69.

Pajaro-Quesada, Rosalba; Betancourt-Campos, Valeria (2007). “¿Qué es una política nacional de información? En: Fernández-Aballi, Isidro (ed.). Building national information policies: experiences in Latin America. Kingston: Unesco, Information for all programme, pp. 86-94. 
http://unesdoc.unesco.org/images/0015/001528/152806m.pdf

Seguí, Rosa (1999). "Maestría en gestión y organización de bibliotecas universitarias: una experiencia de cooperación docente con Nicaragua y El Salvador". BiD: textos universitaris de biblioteconomia i documentació, n. 3 (diciembre). http://bid.ub.edu/O3segui.htm

Shin, Dong-Hee; Kweon, Sang-Hee (2011). "Evaluation of Korean information infrastructure policy 2000-2010: focusing on broadband ecosystem change". Government information quarterly, v. 28, n. 3, pp. 374-387. http://dx.doi.org/10.1016/j.giq.2010.07.009

Soler, Gonzalo-Alejandro (2007). "Evolución y actualidad de las políticas nacionales de información en América Latina y el Caribe". En: Fernández-Aballi, Isidro (ed.). Building national information policies: experiences in Latin America. Kingston: Unesco, Information for all programme, pp. 78-85. http://unesdoc.unesco.org/images/0015/001528/152806m.pdf Souter, David (2005). Towards inclusive knowledge socie- ties: a review of Unesco's action in implementing the WSIS outcomes. Paris: Unesco.

http://unesdoc.unesco.org/images/0018/001878/187832e.pdf

Unesco (1971). Conferencia intergubernamental para el establecimiento de un sistema mundial de información cientifica (Unisist). París: Unesco.

http://unesdoc.unesco.org/images/0000/000006/000630sb.pdf

Unesco (1994). Manifiesto de la Unesco en favor de las bibliotecas públicas.

http://www.unesco.org/webworld/libraries/manifestos/ libraman_es.html

Urbano, Cristóbal (2013). “Inasp: la hora del protagonismo local en la cooperación al desarrollo científico y bibliotecario". Blok de BiD, 24 julio.

http://goo.gl/3EALn1

Wesley-Tanaskovic, Ines (1985). Directrices sobre la política nacional de información: alcance, formulación y aplicación. París: Unesco.

\section{4a Conferencia internacional sobre calidad de revistas de ciencias sociales y humanidades}

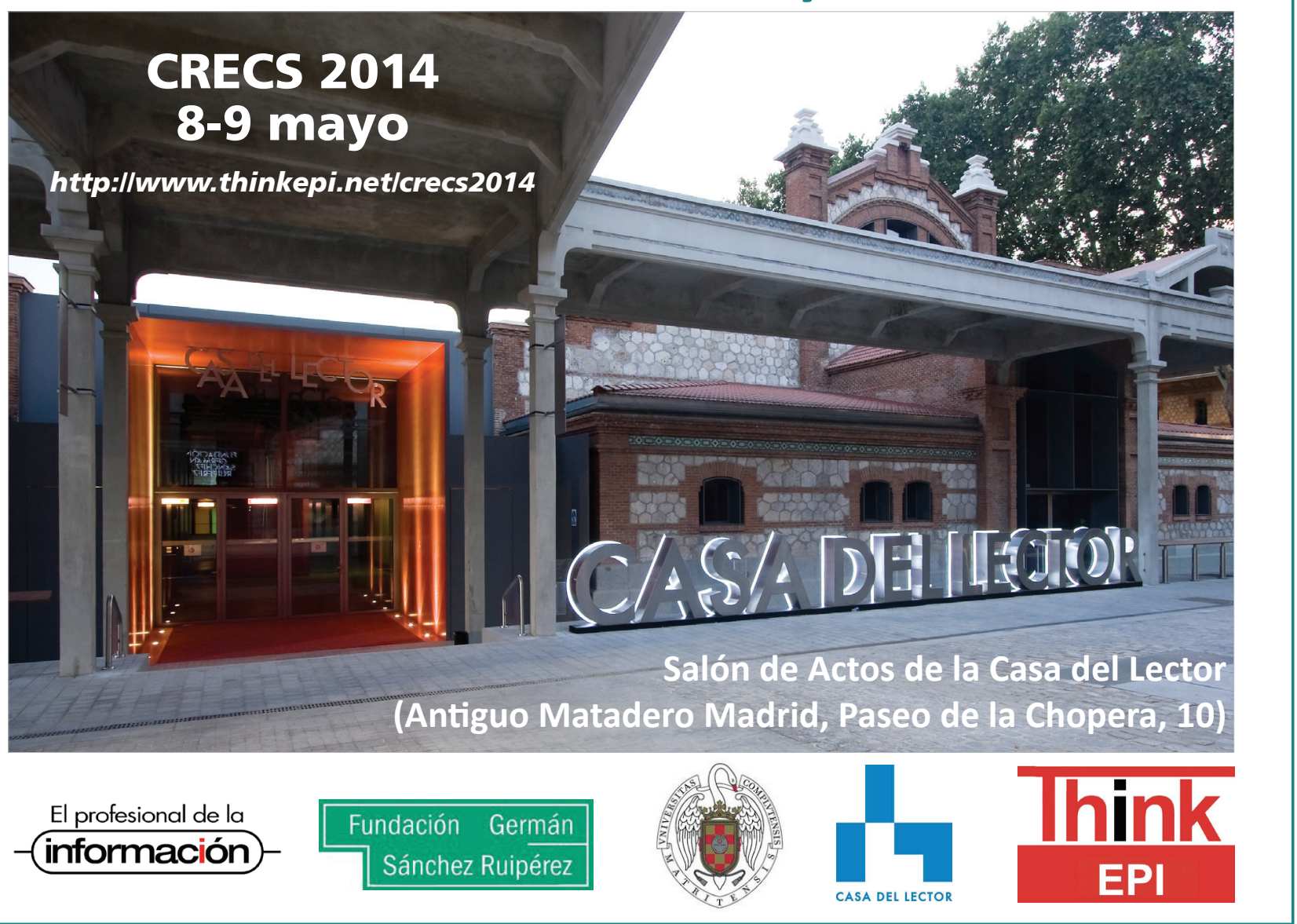

